\title{
ON CONTINUOUS RINGS AND SELF INJECTIVE RINGS( $\left.{ }^{1}\right)$ \\ BY \\ YUZO UTUMI
}

0. Throughout this paper we assume that every ring has a unit element.

A module is called injective if it is a direct summand of every extension module. A ring is said to be left self injective if it is injective as the left module over itself.

The main results we shall show in the present paper are the following:

Let $S$ be a left self injective ring. Then $S / N(S)$ is also left self injective, where $N(S)$ denotes the Jacobson radical of $S$. Any system of orthogonal idempotents of $S / N(S)$ can be lifted to a system of orthogonal idempotents of $S$.

This theorem about orthogonal idempotents can be proved under a somewhat weaker assumption than the left self injectivity of $S$. In fact, it is enough to suppose that $S$ is a ring satisfying the following two conditions:

0.1. Condition. For any left ideal $A$ there is an idempotent $e$ such that $S e$ is an essential extension of $A$.

0.2. Condition. If $S f, f=f^{2}$, is isomorphic to a left ideal $B$, then $B$ also is generated by an idempotent.

We call a ring $S$ which satisfies the above two conditions left continuous.

Then, if $S$ is left continuous, $S / N(S)$ is a (von Neumann) regular ring which is left continuous in the sense that the lattice of principal left ideals of $S$ is upper continuous.

We shall also show some sufficient conditions for a left continuous ring to be left self injective.

\section{Conditions 1.3, 1.4.}

1.1. LemMa. Let $S$ be a ring with Condition 0.2 , and $A$ a left ideal. Let $e$ and $f$ be idempotents such that $S e \cap S(1-f)=0$. If $S e$ is an essential extension of $A$, then Sef is generated by an idempotent, and is an essential extension of Af.

Proof. Since $S e \cap S(1-f)=0$, the right multiplication of $f$ gives an isomorphism of Se onto Sef. Hence $S e f$ is an essential extension of $A f$. By Condition 0.2 Sef is generated by an idempotent.

1.2. THEOREM. Any left continuous ring satisfies the following two conditions:

1.3. Condition. For any idempotent $e$, and for any left ideal $A$ contained in $S e$, there exists an idempotent $f \in$ Se such that $S f$ is an essential extension of $A$.

Received by the editors January 7, 1964.

(1) Supported partly by the NSF grant No. 554-2-184 at the University of Rochester, New York. 
1.4. Condition. If $S g \cap S h=0$ for idempotents $g$ and $h, S g+S h$ is generated by an idempotent.

Proof. By Condition 0.1, $A$ has an essential extension $S p, p=p^{2}$. Since $A \subset S e, A \cap S(1-e)=0$, and hence $S p \cap S(1-e)=0$. By Lemma 1.1, it follows that $S p e$ is generated by an idempotent $f$, and is an essential extension of $A(=A e)$.

Next, let $S g \cap S h=0, g=g^{2}$ and $h=h^{2}$. Then $S(1-g)$ contains an isomorphic image $B$ of $S h$. Condition 0.2 assures that $B$ is generated by an idempotent $q$. Thus, $S g+B$ is generated by the idempotent $g+q-g q$, whence $S g+S h$ also is generated by an idempotent since it is isomorphic to $S g+B$, completing the proof.

Proofs of the following two lemmas are straightforward, and will be omitted.

1.5. LeMMA. Let $v$ be a homomorphism of a ring $S$ into a ring $T$, and suppose that the kernel of $v$ contains no nonzero idempotent. Let $e$ and $f$ be idempotents of $S$. If $S e \subset S f$ and $v(S e)=v(S f)$, then $S e=S f$.

1.6. Lemma. Let $S$ be a ring, and e an idempotent. Then $S e=S f$ and $f=f^{2}$ if and only if $f=e+(1-e) x e$ for some $x \in S$.

The following is a direct consequence of the above known Lemma 1.6.

1.7. Lemma. Let $v$ be a homomorphism of a ring $S$ onto a ring $T$. Let $e$ and $v(x)$ be idempotents of $S$ and $T$ respectively. If $v(S e)=T v(x)$, there is an idempotent $f$ of $S$ such that $S e=S f$ and $v(f)=v(x)$.

Proof. Since $T v(e)=T v(x)$,

$$
v(x)=v(e)+(v(1)-v(e)) v(y) v(e) \text { for some } y .
$$

Set $f=e+(1-e) y e$. Then $S e=S f, f=f^{2}$, and evidently $v(f)=v(x)$, as desired.

2. Under Condition 1.3. If a module $M$ is an essential extension of a submodule $N$, we say that $M$ is essential over $N$, and that $N$ is essential in $M$. Let $w$ be a homomorphism of a module $M_{1}$ into a module $M_{2}$. If $N$ is essential in $M_{2}$, then $w^{-1}(N)$ is essential in $M_{1}$. (See [4].) A left ideal of a ring $S$ is called essential if it is essential in $S$ as a left $S$-module.

We denote the left annihilator of a subset $A$ of a ring by $l(A)$. Similarly, $r(A)$ is the right annihilator of $A$. Following R. E. Johnson we call an element $x$ left singular if $l(x)$ is an essential left ideal. The set of left singular elements of a ring $S$ forms an ideal of $S$, which is called the left singular ideal of $S$. Notation: $Z(S)$. As is easily seen, $Z(S)$ contains no nonzero idempotents. The right singular ideal is defined in the obvious way. (See [4].)

2.1. Lemma. Let $S$ be a ring satisfying Condition 1.3, and e an idempotent. Let $A$ be a left ideal contained in Se. Then Se is essential over $A$ if and only if for any $x \in$ Se there exists an essential left ideal $X$ such that $X x \subset A$. 
Proof. The right multiplication of $x$ gives a homomorphism of $S$ into $S e$. If $A$ is essential in $S e$, the inverse image $X$ of $A$ is essential in $S$. Since $X x \subset A$, this proves the only if part. If part: By Condition 1.3, Se contains an idempotent $f$ such that $S f$ is essential over $A$. Let $S e=S f \oplus B, B$ being a left ideal. Then $B$ is generated by an idempotent $g$. By assumption $G g \subset A$ for some essential left ideal G. $G g \subset A \cap S g \subset S f \cap B=0$. Hence $g \in Z(S)$, and $g=0$. Therefore $S e=S f$, and hence $S e$ is essential over $A$, as desired.

This lemma has the following consequence.

2.2. Lemma. Let $S$ be a ring with Condition 1.3. Let $A$ be an essential left ideal, and e an idempotent. Then Se is an essential extension of Ae.

Proof. Let $x \in S$. The right multiplication of $x$ is an endomorphism of the left $S$-module $S$. Since $A$ is essential, the inverse image $B$ of $A$ is also essential. $B x \subset A$, and hence $B(x e) \subset A e$. Thus, $S e$ is essential over $A e$ by Lemma 2.1, as desired.

We denote by $\bar{A}$ the image of a subset $A$ of a ring $S$ under the canonical mapping of $S$ onto $S / Z(S)$.

2.3. Lemma. Let $S$ be a ring with Condition 1.3, and let $e$ and $f$ be idempotents. Then $\overline{S e} \subset \bar{S} \hat{f}$ if and only if Se is essential over Se $\cap S f$.

Proof. $S \bar{e} \subset \bar{e} \bar{f}$ if and only if $\bar{e} \bar{f}=\bar{e}$. By the definition of $Z(S), \bar{e} \bar{f}=\bar{e}$ if and only if $A(e f-e)=0$ for some essential left ideal $A$. Also $A(e f-e)=0$ if and only if $A e \subset S f$. Suppose first that $A e \subset S f$. Then $A e \subset S e \cap S f \subset S e$. Since $S e$ is essential over $A e$ by Lemma 2.2, $S e$ is an essential extension of $S e \cap S f$. Conversely, if $S e \cap S f$ is essential in $S e$, there is an essential left ideal $A$ such that $A e \subset S e \cap S f$ by Lemma 2.1. Thus $A e \subset S f$, as desired.

3. Under Conditions 1.3, 1.4. As is easily verified Condition 1.4 is equivalent to the following: If $S e \cap S f=0, e=e^{2}$ and $f=f^{2}$, there is an idempotent $g$ such that $S e=S g$ and $S f \subset S(1-g)$.

3.1. Lemma. Let $S$ be a ring with Conditions 1.3 and 1.4. Let $e$ be an idempotent, and $x$ an element such that $\bar{x}=\bar{x}^{2}$. If $\bar{x} \bar{e}=\bar{x}$, there exists an idempotent $f$ such that $f e=f$ and $\bar{f}=\bar{x}$.

Proof. Since the intersection of two essential left ideals is again essential, we can find an essential left ideal $A$ with the properties that $A(x e-x)=0$ and $A\left(x-x^{2}\right)=0$. Hence $A x \subset S e$ and $A x \cap A(1-x)=0$. By Condition 1.3 there are idempotents $f$ and $g$ such that (i) $A x \subset S f \subset S e$ and $A(1-x) \subset S g$, (ii) $S f$ and $S g$ are essential over $A x$ and $A(1-x)$ respectively. Then $S f \cap S g=0$. By Condition 1.4 we may suppose that $S g \subset S(1-f)$. Since $A x \subset S f, A(x f-x)=0$. On the other hand, since $A(1-x) \subset S g \subset S(1-f)$, we have $A(f-x f)=0$. Therefore $A(f-x)=0$, and $\bar{f}=\bar{x}$, as desired. 
Since we have assumed that $S$ has a unit element, the following is a direct consequence of Lemma 3.1.

3.2. Corollary. Let $S$ be a ring with Conditions $1.3,1.4$. If $\bar{x}=\bar{x}^{2}, \bar{x}=\bar{e}$ for some idempotent $e$ of $S$.

The following is slightly different from Lemma 3.1 .

3.3. LEMMA. Let $S$ be a ring satisfying Conditions 1.3 and 1.4. Let $e$ and $f$ be idempotents such that $\bar{e} \bar{f}=\bar{e}$. Then there is an idempotent $g$ with the properties that $g f=g$ and $S=S g \oplus S(1-e)$.

Proof. Since $\bar{e} \bar{f}=\bar{e}, A e \subset S f$ for some essential left ideal $A$. By Condition 1.3, $S f$ contains an idempotent $g$ such that $S g$ is essential over Ae. Since $A e \cap S(1-e)=0$, we have $S g \cap S(1-e)=0$. Now $A e$ is essential in $S e$ by Lemma 2.2, and hence $A e \oplus S(1-e$ ) is essential (in $S e \oplus S(1-e)=S$ ). Thus $S g \oplus S(1-e)$ is also essential. However, it is a direct summand of $S$ by Condition 1.4. It follows therefore that $S=S g \oplus S(1-e)$. Moreover $g f=g$ since $S g \subset S f$, as desired.

3.4. Lemma. Let $S$ be a ring with Condition 1.3, and suppose that $S / Z(S)$ satisfies Condition 1.4. Let $e$ and $f$ be idempotents of $S$. If $\bar{S} \bar{e} \cap \bar{S} \bar{f}=0$, then $\mathrm{Se} \cap S f=0$.

Proof. By Condition 1.4 for $S / Z(S)$ there is an idempotent $\bar{x}$ of $\bar{S}$ such that $S \bar{e}=S \bar{x}$ and $S \bar{f} \subset S(\overline{1}-\bar{x})$. By Lemma 1.7 we can find an idempotent $g$ of $S$ such that $S e=S g$ and $\bar{g}=\bar{x}$. Since $\bar{f} \bar{x}=\overline{0}, \bar{f} \bar{g}=\overline{0}$, and so $A f g=0$ for some essential left ideal $A$. Hence $A f \cap S g=0$, and $A f \cap S e=0$. Af is essential in $S f$ by Lemma 2.2. Therefore $S e \cap S f=0$, as desired.

We need the following in the proof of Lemma 3.6.

3.5. LemMA. Let $S$ be a ring with Conditions 1.3, 1.4, and suppose that $S / Z(S)$ fulfills Condition 1.4. Let $e, f, g$ be idempotents of $S$. If $\bar{S} \bar{e} \oplus \bar{S} \bar{f}=\bar{S} \bar{g}$ and $S e+S f \subset S g$, then $S e \oplus S f=S g$.

Proof. Se $\cap S f=0$ by Lemma 3.4. Hence $S e \oplus S f=S h, h=h^{2}$ by Condition 1.4. Then $S h=S \bar{g}$ and $S h \subset S g$. By Lemma $1.5, S h=S g$, as desired.

3.6. Lemma. Let $S$ be a ring satisfying Conditions 1.3 and 1.4. Suppose that $S / Z(S)$ satisfies Condition 1.4. Let $e$ and $f$ be idempotents such that $\bar{e} \bar{f}=\bar{f} \bar{e}$. Then there is an idempotent $g$ with the properties that $\bar{g}=\hat{f}$ and $e g=g e$.

Proof. Since $\bar{e} \bar{f}\left(=\bar{e}_{1}\right), \bar{e}(\bar{l}-\bar{f})\left(=\bar{e}_{2}\right),(\bar{l}-\bar{e}) \bar{f}\left(=\bar{e}_{3}\right)$ and $(\bar{l}-\bar{e})(\bar{l}-\bar{f})$ ( $=\bar{e}_{4}$ ) are orthogonal idempotents, by Lemma 3.1 we may suppose that each $e_{i}$ is an idempotent such that $e_{1}, e_{2} \in S e, e_{3}, e_{4} \in S(1-e)$. Then $S e=S e_{1} \oplus S e_{2}$ and $S(1-e)=S e_{3} \oplus S e_{4}$ by Lemma 3.5. Let $e=x_{1} e_{1}+x_{2} e_{2}$ and $1-e=x_{3} e_{3}$ 
$+x_{4} e_{4}$, and set $x_{i} e_{i}=f_{i}$ for $i=1,2,3,4$. Then $\left(f_{i}\right)$ is a system of orthogonal idempotents. Set $g=f_{1}+f_{3}$. Evidently $g=g^{2}$ and $e g=g e$. Now $\bar{S} \bar{g}=S \bar{f}_{1}+S \bar{f}_{3}$ $=S \bar{e}_{1}+S \bar{e}_{3}=S \bar{f}$, and similarly $\bar{S}(\overline{1}-\bar{g})=\bar{S}(\overline{1}-\bar{f})$. Therefore $\bar{g}=\bar{f}$, as desired.

4. Left continuous rings. Recall the definition of left continuous rings: A ring $S$ is left continuous if it satisfies Conditions 0.1 and 0.2 .

Thus, in this case $S$ is a ring with Conditions 1.3 and 1.4 by Theorem 1.2. That $S / Z(S)$ also satisfies Condition 1.4 is a consequence of the following. We denote the Jacobson radical of a ring $S$ by $N(S)$.

4.1. LEMmA. If $S$ is a left continuous ring, $Z(S)=N(S)$, and $S / N(S)$ is regular (in the sense of von Neumann).

Proof. First we shall show that $Z(S) \subset N(S)$. Let $x \in Z(S)$. Then $A x=0$ for some essential left ideal $A$. Hence $l(1+x) \cap A=0$, and $l(1+x)=0$. This implies that the right multiplication of $1+x$ is a monomorphism of $S$ into itself. Since $S(1+x)$ is generated by an idempotent by Condition 0.2 , the inverse mapping of the monomorphism is given by the right multiplication of an element $1+y$. Then $(1+x)(1+y)=1$, that is, $x$ is right quasi-regular. Thus, every element of the ideal $Z(S)$ is right quasi-regular, and hence is quasi-regular, which shows that $Z(S) \subset N(S)$.

Next, we shall prove that $S / Z(S)$ is regular. Let $z \in S$, and let $B$ be a maximal left ideal disjoint to $l(z)$. Then it is easy to see that $B \oplus l(z)$ is essential. By Condition $0.1, B$ has an essential extension which is generated by an idempotent. Hence $B$ itself is generated by an idempotent because of the maximality of $B$. Since the right multiplication of $z$ gives an isomorphism of $B$ onto $B z, B z$ also is generated by an idempotent in view of Condition 0.2. Thus the inverse mapping of $B z$ onto $B$ is given by the right multiplication of an element $t$. Then $(B \oplus l(z))(z-z t z)=0$, and so $z-z t z \in Z(S)$, which means that $S / Z(S)$ is a regular ring.

Since every regular ring is semisimple, it follows by the above argument that $Z(S)=N(S)$, completing the proof.

4.2. Corollary. A left continuous ring is regular if (and only if) it is semisimple.

A right continuous ring is defined in the obvious way. A ring which is both left and right continuous is called continuous. The right-left symmetry of Lemma 4.1 assures that the right singular ideal of a right continuous ring coincides with the Jacobson radical. Thus we obtain the following.

4.3. COROLLARY. The left singular ideal of a continuous ring coincides with the right singular ideal.

4.4. LemMA. Let $S$ be a left continuous ring, and let $\left(e_{t}\right)$ be a set of idempotents of $S$. If the sum of $\bar{S} \bar{e}_{t}$ is direct, so is the sum of $S e_{t}$. 
Proof. It is enough to show the lemma in the case $\left(e_{t}\right)$ is finite. Suppose that we have seen that $\sum_{t=1}^{n-1} S e_{t}$ is direct. Then $\sum_{t=1}^{n-1} S e_{t}=S f, f=f^{2}$ by Condition 1.4. $\bar{S} \bar{f} \cap \bar{S} \bar{e}_{n}=0$ by assumption. Thus $S f \cap S e_{n}=0$ by Lemma 3.4 since $\bar{S}$ satisfies Condition 1.4 by Lemma 4.1. Therefore $\sum_{t=1}^{n} S e_{t}$ is direct, which completes the proof by induction.

4.5. LEMMA. Let $S$ be a left continuous ring. Let $\left(e_{t}\right)$ be a set of idempotents of $S$ such that the sum of $S \bar{e}_{t}$ is direct. If $S e, e=e^{2}$, is essential over $\sum S e_{t}$, then $\bar{S} \bar{e}$ is essential over $\sum \bar{S} \bar{e}_{t}$.

Proof. Let $\bar{x}$ be an idempotent of $\bar{S}$ such that $\bar{S} \bar{x} \subset \bar{S} \bar{e}$ and $\bar{S} \bar{x} \cap \sum \bar{S} \bar{e}_{t}=0$. By Lemma 3.1 we may suppose that $x$ is an idempotent in $S e$. In view of Lemma 4.4 the sum $S x+\sum S e_{t}$ is direct. Since $S e$ is essential over $\sum S e_{t}, x=0$, and so $\bar{x}=0$. It follows from this that $\bar{S} \bar{e}$ is an essential extension of $\sum \bar{S} \bar{e}_{t}$, since $\bar{S}$ is regular by Lemma 4.1. This completes the proof.

In [7] we called a regular ring left continuous if the lattice of principal left ideals was upper continuous. By [8, Theorem 2] a regular ring $S$ is left continuous in this sense if and only if it satisfies Condition 0.1. Thus, the definition of left continuity in this paper is consistent with the definition in [7].

4.6. THEOREM. If $S$ is left continuous, then $S / N(S)$ is left continuous regular.

Proof. $S / N(S)(=\bar{S})$ is regular by Lemma 4.1. Let $A$ be a left ideal of $\bar{S}$. We shall show the existence of an idempotent $\bar{e}$ of $\bar{S}$ such that $\bar{S} \bar{e}$ is essential over $A$. By Zorn's lemma we can find a direct sum $\sum \bar{S} \bar{x}_{t}$ of principal left ideals such that $A$ is an essential extension of $\sum \bar{S} \bar{x}_{t}$. Since $\bar{S}$ is regular, we may suppose that each $\bar{x}_{t}$ is an idempotent of $\bar{S}$. Furthermore we may assume that every $x_{t}$ is an idempotent by Corollary 3.2. Let $S e, e=e^{2}$, be an essential extension of $\sum S x_{t}$. Then $\bar{S} \bar{e}$ is essential over $\sum \bar{S} \bar{x}_{t}$ by Lemma 4.5. Let $\bar{x} \in A$. Since $A$ is essential over $\sum \bar{S} \bar{x}_{t}, \bar{S} \bar{x}$ is essential over $\bar{S} \bar{x} \cap \sum \bar{S} \bar{x}_{t}$. Hence $S \bar{x}$ is essential over $\bar{S} \bar{x} \cap \bar{S} \bar{e}$ too, and therefore $\bar{S} \bar{x}=\bar{S} \bar{x} \cap \bar{S} \bar{e}$, whence $\bar{S} \bar{x} \subset \bar{S} \bar{e}$. This implies that $\sum \bar{S}_{\boldsymbol{t}} \subset A \subset \bar{S} \bar{e}$. Since we have seen that $\bar{S} \bar{e}$ is essential over $\sum \bar{S} \bar{x}_{t}, \bar{S} \bar{e}$ is essential over $A$, completing the proof.

Next we shall show that a similar theorem (Theorem 4.8) holds for left self injective rings too.

\subsection{THEOREM. Any left self injective ring is left continuous.}

This is evident by [1, Theorem 57.13].

4.8. THEOREM. If $S$ is left self injective, so is $S / N(S)$.

Proof. By [1, Theorem 57.14] it is enough to see that any left $S$-homomorphism $v$ of a left ideal $A$ of $S$ into $S$ is given by the right multiplication of an element of $\bar{S}$. Since $S$ is left continuous, as in the proof of Theorem 4.6 we can find a system $\left(x_{t}\right)$ 
of idempotents of $S$ such that the direct sum $\sum \bar{S} \bar{x}_{t}$ is essential in $A$. The sum $\sum S x_{t}$ is also direct by Lemma 4.4. For each $t$ the restriction of $v$ on $\bar{S} \bar{x}_{t}$ is given by the right multiplication of an element $\bar{y}_{t}$. The right multiplication of $y_{t}$ gives a homomorphism of $S x_{t}$ into $S$. Combining these homomorphisms for all $t$, we obtain a homomorphism of $\sum S x_{t}$ into $S$, which is given by the right multiplication of an element $y$ since $S$ is left self injective. Then $x_{t} y_{t}=x_{t} y$, and so $v\left(\bar{x}_{t}\right)=\bar{x}_{t} \bar{y}_{t}=\bar{x}_{t} \bar{y}$ for each $t$. Denote by $w$ the difference of $v$ and the right multiplication of $\bar{y}$ on $A$. Since $A$ is essential over $\sum \bar{S} \bar{x}_{i}$, it is easy to see, by Lemma 2.1, that for any $\bar{a} \in A$ there is an essential left ideal $P$ of $\bar{S}$ such that $P \bar{a} \subset \sum \bar{S} \bar{x}_{t}$. Now $w\left(\sum \bar{S} \bar{x}_{t}\right)=0$. Hence $P w(\bar{a}) \subset w\left(\sum \bar{S} \bar{x}_{t}\right)=0$, and therefore $w(A) \subset Z(\bar{S})$. Thus $w(A)$ does not contain any nonzero idempotent, which means that $w(A)=0$ since $S$ is regular. This implies that $v$ is a restriction of the right multiplication of $\bar{y}$, completing the proof.

In the following we shall use the fact that in a regular ring a left ideal has at most one principal essential extension.

4.9. THEOREM. Let $S$ be a left continuous ring, and $e$ an idempotent. Let $\left(\bar{e}_{t}\right)$ be a system of orthogonal idempotents of $S / N(S)$, and suppose that $\bar{S} \bar{e}$ is essential over $\sum \bar{S} \bar{e}_{t}$. Then there is a system $\left(f_{t}\right)$ of orthogonal idempotents of $S$ such that $\bar{e}_{t}=\bar{f}_{t}$ for every $t$, and that $S e$ is an essential extension of $\Sigma S f_{t}$.

Proof. By Lemma 3.1 we may assume that each $e_{t}$ is an idempotent of $S$ contained in $S e$. Let $S p, p=p^{2}$, be an essential extension of $\sum S e_{t}$ such that $S p \subset S e$. By Lemma 4.5, $\bar{S} \bar{p}$ is essential over $\sum \bar{S} \bar{e}_{t}$. Since $\bar{S}$ is regular, it follows that $S \bar{p}=\bar{S} \bar{e}$. Hence $S p=S e$ by Lemma 1.5. Thus $S e$ is essential over $\Sigma S e_{t}$. By Condition 1.3, Se contains an idempotent $g_{t}$ for each $t$ such that $\sum_{u \neq t} S e_{u}$ is essential in $S g_{t} \cdot \bar{S}_{\boldsymbol{t}}$ is essential over $\sum_{u \neq t} \bar{S}_{\bar{e}}$ by Lemma 4.5. By the regularity of $S$, then $S \bar{e}=S \bar{e}_{t} \oplus \bar{S} \bar{g}_{t}$. Since $S e \supset S e_{t}+S g_{t}, S e=S e_{t} \oplus S g_{t}$ by Lemma 3.5. Now $\sum_{u \neq t} \bar{S} \bar{e}_{u} \subset \bar{S}\left(\overline{1}-\bar{e}_{t}\right)$, and so $\bar{S} \bar{g}_{t} \supset \bar{S}\left(\overline{1}-\bar{e}_{t}\right)$. Let $\bar{S}\left(\overline{1}-\bar{e}_{t}\right)=\bar{S} \bar{g}_{t} \oplus \bar{S} \bar{h}_{t}$, $h_{t}=h_{t}^{2}$. We may suppose that $h_{t}=h_{t}^{2}$. Since $\bar{S}=\bar{S} \bar{e}_{t} \oplus \bar{S}\left(\overline{1}-\bar{e}_{t}\right)=\bar{S} \bar{e}_{t} \oplus \bar{S} \bar{g}_{t} \oplus \bar{S} h_{t}$ $=S \bar{e} \oplus S h_{t}$, it follows that $S=S e \oplus S h_{t}$ by Lemma 3.5. Thus, $S=S e_{t} \oplus S g_{t} \oplus S h_{t}$, whence we can find an idempotent $f_{t}$ such that $S e_{t}=S f_{t}$ and $S g_{t} \oplus S h_{t}=S\left(1-f_{t}\right)$. Then $S \bar{e}_{t}=S \bar{f}_{t}$ and $\bar{S}\left(\overline{1}-\bar{e}_{t}\right)=\bar{S} \bar{g}_{t} \oplus S \bar{h}_{t}=\bar{S}\left(\overline{1}-\bar{f}_{t}\right)$. Therefore $\bar{e}_{t}=\bar{f}_{t}$. We have already seen that $S e$ is essential over $\sum S e_{t}$, and hence it is so over $\sum S f_{t}$. If $t \neq u, f_{u} f_{t} \in S f_{u} f_{t}=S e_{u} f_{t} \subset S g_{t} f_{t} \subset S\left(1-f_{t}\right) f_{t}=0$. Thus $\left(f_{t}\right)$ is a system of orthogonal idempotents, which completes the proof.

As a direct consequence of this we have

4.10. CoROLlaRY. Let $S$ be a left continuous ring, and $\left(\bar{e}_{t}\right)$ a system of orthogonal idempotents of $S / N(S)$. Then there exists a system $\left(f_{t}\right)$ of orthogonal idempotents of $S$ such that $\bar{e}_{t}=\bar{f}_{t}$ for every $t$.

Proof. Let $\bar{S} \bar{e}$ be the essential extension of $\sum \bar{S} \bar{e}_{t}$, where $\bar{e}=\bar{e}^{2}$. We may suppose that $e$ is an idempotent by Corollary 3.2. Thus the corollary follows by Theorem 4.9. 
4.11. COROLlaRY. In Theorem 4.9 if we assume moreover that $\bar{e} \bar{e}_{t}=\bar{e}_{t}$ for each $t$, then we can find a system $\left(g_{t}\right)$ of orthogonal idempotents of $S$ such that $S e$ is essential over $\sum S g_{t}$, and $\bar{e}_{t}=\bar{g}_{t}$ and $e g_{t}=g_{t}$ for each $t$.

Proof. Set $g_{t}=e f_{t}$, where $f_{t}$ is the idempotent in Theorem 4.9. Then $S g_{t}=S f_{t}$, and the corollary follows immediately from Theorem 4.9.

4.12. Corollary. Let $S$ be a left continuous ring, and e an idempotent. Let $\left(\bar{e}_{i}\right)$ be a finite system of orthogonal idempotents of $S / N(S)$ such that $\bar{e}=\sum \bar{e}_{i}$. Then we can choose a system $\left(g_{i}\right)$ of orthogonal idempotents of $S$ in such a way that $\bar{e}_{i}=\bar{g}_{i}$ for every $i$ and $e=\sum g_{i}$.

Proof. Let $\left(g_{i}\right)$ be the system in Corollary 4.11, and set $g=\Sigma g_{i}$. Since $S e$ is essential over $\sum S g_{i}=S g, S e=S g$, and so $e g=e$. Since moreover $e g=g$ by Corollary 4.11, we have $e=g$, as desired.

\section{Continuous rings.}

5.1. THEOREM. Let $S$ be a continuous ring. Then $S$ satisfies the following.

5.2. Condition. $x y=1$ implies $y x=1$, that is, any one-sided inverse element is two-sided.

This theorem is a consequence of the following.

5.3. Lemma. If a ring $S$ fulfills Condition 0.1 and its right-left symmetry, then $S$ satisfies Condition 5.2.

Proof. Suppose that $x y=1$ and $y x \neq 1$ for some $x, y \in S$. Then $S$ contains a system $\left(e_{i j} ; i, j=1,2, \cdots\right)$ of matrix units of countably infinite degree by a theorem of Jacobson. (See [2].) Let $S e, e=e^{2}$, be an essential extension of $\Sigma S e_{i i}$. We may suppose that $e e_{i j}=e_{i j}$ for every $i, j$ taking $e e_{i j}$ instead of $e_{i j}$ if necessary. Let $f S$ be an essential extension of $\sum_{j>1}\left(e_{1 j}+e_{j j}\right) S, f$ being an idempotent.

First assume that $e \neq f e$. Then $0 \neq(1-f) e \in S e$, and hence there is $x$ such that $0 \neq x(1-f) e \in \sum_{i=1}^{n} S e_{i i}$ for some finite $n$. Then $x(1-f) e e_{n+1, n+1}=0$ by the orthogonality of $\left(e_{i i}\right)$. On the other hand, $(1-f)\left(e_{1 j}+e_{j j}\right)=0$ for each $j>1$ by the definition of $f$. Hence

$$
x(1-f) e_{1, n+1}=x(1-f)\left(e_{1, n+1}+e_{n+1, n+1}\right)-x(1-f) e e_{n+1, n+1}=0 .
$$

Thus, $x(1-f) e_{1, n+1}=0$, and so $x(1-f) e_{1, i}=0$ for every $i$. In particular, $x(1-f) e_{11}=0$. For any $j>1$ we have

$$
x(1-f) e_{j j}=x(1-f)\left(e_{1 j}+e_{j j}\right)-x(1-f) e_{1 j}=0 .
$$

Therefore $x(1-f) e_{i i}=0$ for every $i$. Now denote by $A$ the set of elements $y$ in $S e$ such that $y e_{i i}=0$ for every $i$. Then $x(1-f) e \in A$. $A$ is a left ideal contained in $S e$, 
and is disjoint to $\sum S e_{i i}$. Since $S e$ is essential over $\sum S e_{i i}$, it follows that $A=0$, whence $x(1-f) e=0$, a contradiction.

Next, suppose that $e=f e$. Then $e_{11}=e e_{11}=f e e_{11} \in f S$. Hence $0 \neq e_{11} z \in \sum_{j>1}\left(e_{1 j}+e_{j j}\right) S$ for some $z$. Let $e_{11} z=\sum_{j=2}^{m}\left(e_{1 j}+e_{j j}\right) z$. Since the sum $\sum e_{i i} S$ is direct, we have $e_{11} z-\sum_{j=2}^{m} e_{1 j} z_{j}=0$ and $e_{j j} z_{j}=0$ for $j=2, \cdots, m$, and therefore $e_{11} z=0$, a contradiction. This completes the proof.

The following is almost obvious.

5.4. Lemma. Let $S$ be a ring with Condition 5.2. Let $e, f$ be idempotents such that $S e \subset S f$ and $S e \simeq S f$. Then $S e=S f$.

Proof. Set $g=1-f+f e$. Then $g=g^{2}$, and $S g=S e \oplus S(1-f) \simeq S$. Hence $1=x y$ and $y x=g$ for some $x, y$. Thus $g=1$, and $S e=S f$, as desired.

As is known the lattice of principal left ideals of a left continuous regular ring is complete, and hence any annihilator left ideal is generated by an idempotent. (See [7, Lemma 1].) If a principal left ideal of a regular ring is an ideal, then it is generated by a central idempotent. (See [5, Lemma 2.6, II].)

We shall use the following in the proof of Theorem 5.6.

5.5. LEMMA. Let $S$ be a left continuous ring, and e, $f$ idempotemts. Suppose that $S g \neq S h$ for any nonzero idempotents $g$ and $h$ such that $g=g e$ and $h=h f$. Then there exist idempotents $p, q, s$ and $t$ with the following properties: (1) $S p \oplus S(1-e)=S$ and $S q \oplus S(1-f)=S$; (2) $p s=p$ and $q t=q$; (3) $s t$ $=t s=0$; (4) $\bar{s}$ and $\bar{t}$ are central in $\bar{S}$.

Proof. Let $\bar{x} \in \bar{e} \bar{S} \bar{f}$. Then the right multiplication of $\bar{x}$ gives a homomorphism $v$ of $\bar{S} \bar{e}$ into $\bar{S} \bar{f}$. The kernel $K$ of $v$ is generated by an idempotent. Let $\bar{S} \bar{e}=K \oplus \bar{S} \bar{g}, \bar{g}=\bar{g}^{2}$. We may suppose that $g=g^{2} \in S e$. The image of $v$ is also generated by an idempotent $h$. We assume that $h=h^{2} \in S f$. Evidently $S \bar{g} \simeq S h$. Since $N(S)$ is the Jacobson radical of $S$, it follows that $S g \simeq S h$. Hence $h=0$ by assumption. Thus, $v=0$, and $\bar{x}=\overline{0}$, whence $\bar{e} \bar{S} \bar{f}=\overline{0}$.

Let $\bar{S} \bar{s}=l(r(\bar{e} \bar{S}))$ and $\bar{t} \bar{S}=r(l(\overline{S f})), \bar{s}$ and $\bar{t}$ being idempotents. Then $\bar{s}, \bar{t}$ are central and orthogonal, proving (4). By Lemma 3.6 we may assume that $s, t$ are idempotents of $S$ such that $s t=t s$. Thus, $s t=(s t)^{2} \in N(S)$, and so $s t=0$, proving (3). By Lemma 3.3, since $S \bar{e} \subset S \bar{s}$, we can find an idempotent $p$ such that $p s=p$ and $S=S p \oplus S(1-e)$. Similarly we have an idempotent $q$ such that $q t=q$ and $S=S q \oplus S(1-f)$, proving (1) and (2). This completes the proof of the lemma.

5.6. THEOREM. Let $S$ be a left self injective ring satisfying Condition 5.2. Then any isomorphism between two left ideals of $S$ can be extended to an automorphism of the left $S$-module $S$.

Proof. By Zorn's lemma there is a maximal isomorphism $v$ between left ideals $A$ and $B$ of $S$ which contains the given isomorphism in the sense of graph. 
Let $S(1-e), e=e^{2}$, and $S(1-f), f=f^{2}$, be essential over $A$ and $B$ respectively. Then by [1, Theorem 57.13] $v$ can be extended to an isomorphism of $S(1-e)$ and $S(1-f)$. Thus, $A=S(1-e)$ and $B=S(1-f)$ by the maximality of $v$. Se and $S f$ do not contain any mutually isomorphic left ideals. Hence, by Lemma 5.5 we have idempotents $p, q, s$ and $t$ with the properties (1), (2), (3) and (4) in Lemma 5.5. $S s \cap S t=0$ by (3), and so $S p \cap S t=0$ by (2). In view of (1) this means that $S(1-e)$ contains an isomorphic image of $S t$. Since $S(1-e) \simeq S(1-f)$, $S(1-f)$ also contains an isomorphic image $S u$ of $S t$. By Condition 0.2 we may suppose that $u=u^{2}$. Hence $\bar{S} \bar{t} \simeq S \bar{u}$. Now $S \bar{t}$ is an ideal by (4). Thus, $S \bar{t} \supset S \bar{u}$. Since $S$ satisfies Condition 5.2, so does $S=S / N(S)$. Therefore $S \bar{t}=S \bar{u}$ by Lemma 5.4. By Lemma 2.3, $S t$ is an essential extension of $S t \cap S u$. However, $S q \subset S t$ by (2), and also $S q \cap S u \subset S q \cap S(1-f)=0$ by (1). Thus, $S q=0$, and so $S(1-f)=S$ by (1), whence $S \simeq S(1-e)$. By Lemma $5.4, S=S(1-e)$. Therefore $v$ is an automorphism of the left $S$-module $S$, completing the proof.

6. Join of orthogonal idempotents. Let $\left(e_{t}\right)$ be a system of orthogonal idempotents of a ring $S$. We call an idempotent $e$ a join of $\left(e_{t}\right)$ if $S e$ and $e S$ are essential over $\Sigma S e_{t}$ and $\sum e_{t} S$ respectively.

6.1. THEOREM. If an idempotent $e$ is a join of a system of orthogonal idempotents $\left(e_{t}\right)$ of a ring $S$, we have $S(1-e)=l\left(\left(e_{t}\right)\right)$ and $(1-e) S=r\left(\left(e_{t}\right)\right)$. $e$ is the only join of $\left(e_{t}\right)$.

Proof. Since $e_{t} S \subset e S$ for every $t, l\left(\left(e_{t}\right)\right) \supset S(1-e)$. Now $l\left(\left(e_{t}\right)\right) \cap \sum S e_{t}=0$, and hence $l\left(\left(e_{t}\right)\right) \cap S e=0$. Thus $l\left(\left(e_{t}\right)\right)=S(1-e)$. Similarly $r\left(\left(e_{t}\right)\right)=(1-e) S$. In case $f$ is also a join of $\left(e_{t}\right)$, then $S(1-e)=S(1-f)$ and $(1-e) S=(1-f) S$. It follows from these that $e=f$, as desired.

6.2. Corollary. If $\left(e_{t}\right)$ is a system of orthogonal central idempotents, and if $e$ is its join, then e also is central.

Proof. In this case $l\left(\left(e_{t}\right)\right)=r\left(\left(e_{t}\right)\right)$, and hence $S(1-e)=(1-e) S$ by Theorem 6.1. This implies that $1-e$, and hence $e$ also is central.

6.3. THEOREM. Let $S$ be a right self injective ring. Let $e$ be the join of a system $\left(e_{t}\right)$ of orthogonal idempotents. Then $S e$ is isomorphic to the complete direct sum $D=\left\{\left[x_{t} e_{t}\right]: x_{t} \in S\right\}$ of Se $e_{t}$ by the correspondence $v: x(\in S e) \rightarrow\left[x e_{t}\right](\in D)$.

Proof. It is evident that $v$ is a homomorphism. If $x e_{t}=0$ for every $t, x \in S(1-e)$ by Theorem 6.1, and hence $x \in S(1-e) \cap S e=0$, which shows that $v$ is a monomorphism. Let $\left[x_{t} e_{t}\right] \in D$. The left multiplication of $x_{t}$ gives a homomorphism of $e_{t} S$ into $S$. Combining these homomorphisms for all $t$ we obtain a homomorphism of $\sum e_{t} S$ into $S$, which is given by the left multiplication of an element $x$ in view of the right self injectivity of $S$. $x e e_{t}=x e_{t}=x_{t} e_{t}$ for every $t$. Hence $v(x e)=\left[x_{t} e_{t}\right]$, and therefore $v$ is an isomorphism, as desired. 
6.4. THEOREM. Let $S$ be a continuous ring, and $\left(e_{t}\right)$ a system of orthogonal idempotents. Then there exists the join of $\left(e_{i}\right)$.

Proof. (1) Suppose first that $S$ is continuous regular. Then the lattice of principal left ideals of $S$ is complete, and the join $\bigcup S e_{t}$ is essential over $\Sigma S e_{t}$. As is easily seen $\left(\Sigma S e_{t}\right) \cap\left(\bigcap S\left(1-e_{t}\right)\right)=0$, and hence $\left(\bigcup S e_{t}\right) \cap\left(\bigcap S\left(1-e_{t}\right)\right)$ $=0$. (Note that the meet $\bigcap S\left(1-e_{t}\right)$ is the set-theoretical intersection of $S\left(1-e_{t}\right)$ since the lattice is complete. See [7, Lemma 1].) Similarly $\left(\bigcup e_{t} S\right) \cap\left(\bigcap\left(1-e_{t}\right) S\right)$ $=0$. Taking the left annihilators of both sides of this relation, we have $\left(\bigcap S\left(1-e_{t}\right)\right)+\left(\bigcup S e_{t}\right)=S$. Therefore $\left(\bigcup S e_{t}\right) \oplus\left(\bigcap S\left(1-e_{t}\right)\right)=S$, whence there exists an idempotent $e$ such that $S e=\bigcup S e_{t}$ and $S(1-e)=\bigcap S\left(1-e_{t}\right)$. It follows from the last relation that $e S=\bigcup e_{t} S$, where $\bigcup e_{t} S$ is essential over $\sum e_{t} S$ by assumption. Thus, $e$ is the join of $\left(e_{t}\right)$, as desired.

(2) To see the theorem in the general case, let $S e, e=e^{2}$, be essential over $\sum S e_{t}$, and $f S, f=f^{2}$, essential over $\sum e_{t} S$. Then $\bar{S} \bar{e}$ and $\bar{f} S$ are essential over $\sum \bar{S} \bar{e}_{t}$ and $\sum \bar{e}_{t} S$ respectively by Lemma 4.5 . Let $\bar{g}$ be the join of $\left(\bar{e}_{t}\right)$, which exists by (1). $\bar{S} \bar{e}=\bar{S} \bar{g}$ and $\bar{f} \bar{S}=\bar{g} S$ by the regularity of $S$. Hence there are idempotents $r$ and $s$ such that $S e=S r, \bar{r}=\bar{g}$, and $f S=s S, \bar{s}=\bar{g}$ by Lemma 1.7. Thus $\bar{r}=\bar{s}$, and $\overline{1}-\bar{r}=\overline{1}-\bar{s}$. $S(1-s)$ is essential over $S(1-s) \cap S(1-r)$ by Lemma 2.3, and so $S(1-s) \cap S r=0$. By Lemma 1.1, Srs is generated by an idempotent, and moreover $S r s$ is essential over $\sum S e_{t}$. Now $\sum S \bar{e}_{t} \bar{s}=\sum S \bar{e}_{t}$ since $\bar{e}_{t} \bar{s}=\bar{e}_{t} \bar{g}$ $=\bar{e}_{t}$. Hence $\bar{S} \bar{r} \bar{s}$ is essential over $\sum S \bar{e}_{t}$ by Lemma 4.5. On the other hand, $S \bar{s}$ is also essential over $\sum \bar{S}_{\bar{t}}$ since $\bar{S} \bar{s}=\bar{S} \bar{g}=S \bar{e}$ and $S \bar{e}$ is essential over $\sum S \bar{e}_{t}$. Therefore $\bar{S} \bar{r} \bar{s}=S \bar{s}$. Since we have seen that $S r s$ is generated by an idempotent, $S r s=S s$ by Lemma 1.5. $S s=S r s=S(r-r(1-s)) \subset S r \oplus S(1-s)$, and hence $S=S r \oplus S(1-s)$. There exists an idempotent $p$ such that $S r=S p$ and $S(1-s)$ $=S(1-p)$. Then $S e=S r=S p$ and $f S=s S=p S$, whence $p$ is the join of $\left(e_{t}\right)$, completing the proof.

6.5. THEOREM. Let $S$ be a continuous ring, and $e$ an idempotent. Let $\left(\bar{e}_{t}\right)$ be a system of orthogonal idempotents of $S / N(S)$, and suppose that $\bar{e}$ is the join of $\left(\bar{e}_{t}\right)$. Then there is a system $\left(f_{t}\right)$ of orthogonal idempotents of $S$ such that $\bar{e}_{t}=\bar{f}_{t}$ for every $t$, and that $e$ is the join of $\left(e_{t}\right)$.

Proof. By Corollary 4.11 we can find a system $\left(f_{t}\right)$ of orthogonal idempotents of $S$ such that $S e$ is essential over $\sum S f_{t}$, and $\bar{f}_{t}=\bar{e}_{t}$ and $e f_{t}=f_{t}$ for every $t$. Since $e S \supset \sum f_{t} S$, there is an essential extension $f S$ of $\sum f_{t} S$ such that $e S \supset f S$ and $f=f^{2}$ by Condition 1.3. $\bar{f} \bar{S}$ is essential over $\sum \bar{f}_{t} S\left(=\sum \bar{e}_{t} S\right)$ by Lemma 4.5. By assumption $\bar{e} \bar{S}$ is also essential over $\sum \bar{e}_{1} S$. Hence $\bar{f} S=\bar{e} S$. Therefore $f S=e S$ by Lemma 1.5, whence $e$ is the join of $\left(f_{t}\right)$, as desired.

7. Sufficient conditions. In this section we shall consider some sufficient conditions for a left continuous ring to be left self injective. 
7.1. THEOREM. Let $S$ be a left continuous ring, and suppose that the unit element 1 is a sum of orthogonal idempotents $e_{i}, i=1,2, \cdots, n, n$ being greater than 1. If each $S\left(1-e_{i}\right)$ contains an isomorphic image of $S e_{i}$, then $S$ is left self injective.

To see this we need the following two lemmas.

7.2. Lemma. Let $S$ be a left continuous ring, and let $S e, e=e^{2}$, be an essential extension of a left ideal $A$. Let $v$ be a homomorphism of $A$ into $S$. $S u p p o s e$ that $S e \cap S f=0$ and $v(A) \subset S f$ for an idempotent $f$. Then $v$ can be extended to a homomorphism of Se into $S f$.

Proof. As $S e \oplus S f$ is generated by an idempotent, we may suppose that $e$, $f$ are orthogonal. Set $G=\{a+v(a): a \in A\}$. Then $G \subset S(e+f)$, and hence $S(e+f)$ contains an idempotent $g$ such that $S g$ is essential over $G$ by Condition 1.3. It is easy to see that $G \cap S(1-e)=0$, whence $S g \cap S(1-e)=0$. Since $G e=A$, it follows by Lemma 1.1 that $S g e$ is essential over $A$, and is generated by an idempotent. Hence $S e=S g e$. Let $e=x g e$, and set $x g f=t$. Then $a+a t$ $=a e+a t=a x g e+a x g f=a x g \in S g$ for any $a \in A$. On the other hand, $a+v(a) \in G \subset S g$. Therefore $a t-v(a) \in S g \cap S f \subset S g \cap S(1-e)=0$, and hence $v$ is a restriction of the right multiplication of $t$. Since $\operatorname{Set}=\operatorname{Sex} g f \subset S f$, this completes the proof.

In the next lemma we shall consider the following property of a submodule $N$ of a module $M$ :

7.3. Property. Any homomorphism defined on a submodule of $N$, and having the values in $M$ can be extended to a homomorphism of $N$ into $M$.

7.4. Lemma. Let $M$ be a module, and $N_{1}, N_{2}$ be submodules of $M$ such that $N_{1} \cap N_{2}=0$. If both $N_{1}$ and $N_{2}$ have Property 7.3, so does $N_{1}+N_{2}$.

Proof. Let $K$ be a submodule of $N_{1} \oplus N_{2}$, and $v$ a homomorphism of $K$ into $M$. By assumption the restriction of $v$ over $K \cap N_{2}$ can be extended to a homomorphism $p$ of $N_{2}$ into $M$. As $v$ and $p$ coincide on $K \cap N_{2}$, there is a homomorphism $q$ of $\left(K+N_{2}\right) \cap N_{1}$ into $M$ given by $q(k+n)=v(k)+p(n)$ for all $k \in K, n \in N_{2}$ with $k+n \in N_{1}$. By assumption $q$ can be extended to a homomorphism $r$ of $N_{1}$ into $M$. Denote by $e_{1}$ and $e_{2}$ the projections of $N_{1} \oplus N_{2}$ to $N_{1}$ and $N_{2}$ respectively. Let $x \in K$. Then $x=e_{1}(x)+e_{2}(x)$, and hence $e_{1}(x)$ $=x-e_{2}(x) \in N_{1} \cap\left(K+N_{2}\right)$. Thus, $r e_{1}(x)=q e_{1}(x)=v(x)-p e_{2}(x)$, and therefore $v(x)=\left(r e_{1}+p e_{2}\right)(x)$, where $r e_{1}+p e_{2}$ is a homomorphism of $N_{1} \oplus N_{2}$ into $M$, as desired.

Proof of Theorem 7.1. By virtue of Lemma 7.4 it is enough to show that any homomorphism $w$ of a left ideal $A$ of $S$ into $S$ can be extended to a homomorphism of $S e_{i}$ into $S$ if $A \subset S e_{i}$. Let $S e, e=e^{2}$, be an essential extension of $A$ such 
that $S e \subset S e_{i}$, and let $e=e_{i} e$. Then $S\left(1-e_{i}\right) \subset S(1-e)$, and hence $S(1-e)$ contains an isomorphic image of $S e$ by assumption, which is generated by an idempotent $f$ by Condition 0.2 . There are $p, q$ such that $p q=e, p=p f$. The mapping $a \rightarrow w(a)(1-e)$ for $a \in A$ is a homomorphism of $A$ into $S(1-e)$, and hence is extended to a homomorphism of $S e$ into $S(1-e)$ by Lemma 7.2. Thus it is given by the right multiplication of an element $x$. Similarly the mapping $a \rightarrow w(a) p(a \in A)$ is also obtained by the right multiplication of an element $y$. Then $a(x+y q)=w(a)(1-e)+w(a) p q=w(a)$ for any $a \in A$, as desired.

A ring $S$ is said to be of order $n$ if the unit element is the sum of orthogonal idempotents $e_{i}, i=1, \cdots, n$, such that $S e_{j} \simeq S e_{i k}$ for any $j, k$. (See [5, Chapter 3, II].)

7.5. Corollary. A ring of order $n, n>1$, is left continuous (if and) only if it is left self injective.

A ring is called strongly regular if for any element $x$ there is an element $y$ such that $x^{2} y=x$. A regular ring is strongly regular if and only if it contains no nonzero nilpotent element, that is, it is of (nilpotency) index 1 .

7.6. THEOREM. Let $S$ be a left continuous ring, and suppose that $S / N(S)$ does not contain any nonzero ideal which is strongly regular as a ring. Then $S$ is left self injective.

To see this theorem we prepare the following.

7.7. Lemma. Let $S$ be a left self injective regular ring with no nonzero strongly regular ideal. Then there is a system of orthogonal idempotents $e_{1}, e_{2}, e_{3}$ such that (1) $1=e_{1}+e_{2}+e_{3}$, (2) $S e_{1} \simeq S e_{2}$, and (3) $S e_{1} \oplus S e_{2}$ contains an isomorphic image of $\mathrm{Se}_{3}$.

Proof. By [7, Lemma 4] $S=S e_{1} \oplus S e_{2} \oplus S e_{3}$, where $e_{i}$ 's are orthogonal idempotents such that $S e_{1} \simeq S e_{2}$, and that $S e_{3}$ does not contain any direct sum of two nonzero mutually isomorphic left ideals. If $S e_{1} \oplus S e_{2}$ contains no isomorphic image of $\mathrm{Se}_{3}$, then $\mathrm{Se}_{3}$ contains a nonzero central idempotent $z$ by [7, Lemma 3]. $S z$ does not contain any direct sum of two mutually isomorphic nonzero left ideals. Hence $S z$ contains no nonzero nilpotent element by [7, (S1)]. Thus $S z$ is strongly regular, contradicting the assumption. Therefore $S e_{1} \oplus S e_{2}$ contains an isomorphic image of $\mathrm{Se}_{3}$, as desired.

7.8. LEMMA. Let $S$ be a left continuous ring, and suppose that $S / N(S)$ satisfies the assumption of Theorem 7.1. Then $S$ itself also satisfies the assumption.

Proof. $\overline{1}=\sum_{i=1}^{n} \bar{e}_{i}$, where $\bar{e}_{i}$ 's are orthogonal idempotents, and $n>1$. By assumption each $\bar{S}\left(\overline{1}-\bar{e}_{i}\right)$ contains an isomorphic image $S \bar{f}_{i}$ of $S \bar{e}_{i}$. Since $S$ is regular by Lemma 4.1 we may suppose that $\tilde{f}_{i}$ is an idempotent for each $i$. By Corollary 4.12 we may assume that $e_{i}$ 's are orthogonal idempotents with 
$1=\sum_{i=1}^{n} e_{i}$. Moreover, by Lemma 3.1 we may assume also that each $f_{i}$ is an idempotent contained in $S\left(1-e_{i}\right)$. Since $N(S)$ is the Jacobson radical of $S$, the assumption $\bar{S} \bar{e}_{i} \simeq \bar{S} \bar{f}_{i}$ implies $S e_{i} \simeq S f_{i}$, completing the proof.

Proof of Theorem 7.6. $S / N(S)$ is left continuous by Theorem 4.6. Hence $S / N(S)$ is left self injective by [7, Corollary to Theorem 3]. Thus, $S / N(S)$ satisfies the assumption of Theorem 7.1 by Lemma 7.7, whence $S$ also satisfies the assumption by Lemma 7.8. Therefore $S$ is left self injective by Theorem 7.1.

7.9. TheOREM. A (left or right) primitive ring $S$ is left self injective if (and only if) it is left continuous.

Proof. $S$ is then semisimple, and hence is regular by Corollary 4.2. If $S$ is strongly regular, it is a division ring since every idempotent of a strongly regular ring is central. If $S$ is not strongly regular $S$ does not contain any nonzero strongly regular ideals by [7, Corollary to Theorem 4$]$. Thus, it is left self injective by Theorem 7.6. This completes the proof.

As is known a ring with minimum conditions for left ideals and for right ideals is left self injective if and only if it is right self injective. In this case the ring is called a quasi-Frobenius ring. (See [1, Theorem 58.6].)

7.10. TheоRем. A ring $S$ with minimum conditions for left and right ideals is quasi-Frobenius if (and only if) it is continuous.

Proof. If $A$ is a minimal left ideal of $S$, then $A S=\Sigma_{x \in S} A x$ is a sum of minimal left ideals isomorphic to $A$. This fact shows that every minimal ideal of $S$ is contained in the left socle $P$, and also that any homogeneous componentl of $P$ is an ideal.

Denote by $T$ the sum of minimal ideals, that is, the socle of the $(S, S)$-module $S$. Then $P \supset T$ as we have seen. We shall show that $P=T$. Let $B$ be a nonzero ideal contained in a homogeneous component $H$ of $P$, and $A_{1}$ a minimal left ideal contained in $B$. Let $H=A_{1} \oplus \cdots \oplus A_{k}$, each $A_{i}$ being a minimal left ideal. By Condition $0.1, A_{i}$ has an essential extension $S e_{i}, e_{i}=e_{i}^{2} . S e_{1} \cap S e_{j}=0$ for every $j>1$. Now $A_{1}$ is isomorphic to $A_{j}$, and the isomorphism can be extended to a homomorphism of $S e_{1}$ into $S e_{j}$ by Lemma 7.2, and hence is given by the right multiplication of an element of $S$. Thus, $A_{j} \subset A_{1} S \subset B$ for any $j>1$, whence $B=H$. This shows that each homogeneous component $H$ of $P$ is a minimal ideal. Therefore $T \supset P$, and $T=P$. Similarly we can show that the right socle $Q$ of $S$ also coincides with $T$. Thus, we have $P=Q$.

Let $e$ be a primitive idempotent. Then by Condition $1.3, S e$ is essential over every nonzero left ideal contained in it. Hence $\operatorname{Se} \cap P$ is a minimal left ideal. Since $Q e=P e=P \cap S e, Q e$ is minimal. Right-left symmetrically $e P$ is a minimal right ideal. Thus $S$ satisfies (ii) of [1, Theorem 58.6] by [1, Lemma 58.3]. Therefore $S$ is quasi-Frobenius, completing the proof. 
7.11. Remark. There is a left continuous ring which is not left self injective. See [7, Example 3]. The ring may be commutative, regular, and may have a minimal ideal.

8. Total matrix rings. Let $S$ be a ring, and denote the total matrix ring of degree $n$ over $S$ by $S_{n}$. Let $\left(e_{i j}\right)$ be a system of matrix units: $S_{n}=\sum S e_{i j}$. The following two properties are well known:

8.1. $S_{n}$ is the endomorphism ring of the left module $e_{11} S_{n}$ over $e_{11} S_{n} e_{11}$ in the natural way.

8.2. The lattice $L$ of submodules of the left $e_{11} S_{n} e_{11}$-module $e_{11} S_{n}$ and the lattice $M$ of left ideals of $S_{n}$ are isomorphic under the following mutually reciprocal mappings:

$$
\begin{aligned}
& p: A(\in L) \rightarrow S_{n} A(\in M) . \\
& q: B(\in M) \rightarrow e_{11} B(\in L) .
\end{aligned}
$$

We shall now show the following.

8.3. THEOREM. A ring $S$ is left self injective if and only if so is the total matrix ring $S_{n}$.

Proof. Suppose first that $S$ is left self injective. Let $B$ be a left ideal of $S_{n}: B \in M$. By assumption $e_{11} S_{n} e_{11}(\simeq S)$ is left self injective, and so is the $e_{11} S_{n} e_{11}$-module $e_{11} S_{n}$ by [1, Theorem 57.3]. Hence $e_{11} B(\in L)$ has an essential extension $G$ which is a direct summand of the module $e_{11} S_{n}$. (See [1, Theorem 57.13, 57.9].) By 8.1 there is an idempotent $e$ such that $G=e_{11} S_{n} e$. Then $S_{n} e(=p(G))$ is essential over $B\left(=p q(B)=p\left(e_{11} B\right)\right)$ in view of 8.2. This proves that $S_{n}$ satisfies Condition 0.1 .

To see that $S_{n}$ satisfies Condition 0.2 too, suppose that a left ideal $C$ of $S_{n}$ is isomorphic to $S_{n} f, f=f^{2}$. Then there is an element $x \in S_{n}$ such that $S_{n} f x=C$ and $l(x) \cap S_{n} f=0$. Thus the right multiplication of $x$ gives an isomorphism of $e_{11} S_{n} f$ onto $e_{11} C$. Since $e_{11} S_{n} f$ is a direct summand of the injective module $e_{11} S_{n}$, it is also injective, and hence $e_{11} C$ too is injective. Therefore $e_{11} C$ is a direct summand of $e_{11} S_{n}$, whence $e_{11} C=e_{11} S_{n} g$ for some idempotent $g \in S_{n}$. It follows then that $C=p q(C)=p\left(e_{11} C\right)=p\left(e_{11} S_{n} g\right)=S_{n} g$. This shows that $S_{n}$ fulfills Condition 0.2. Thus, $S_{n}$ is left continuous. Since $S_{n}$ is of order $n$, if $n>1, S_{n}$ is left self injective by Corollary 7.5.

Suppose next that $S_{n}$ is left self injective, and we shall show that $S$ is left self injective. Let $D$ be a left ideal of $e_{11} S_{n} e_{11}$, and $v$ an $e_{11} S_{n} e_{11}$-homomorphism of $D$ into $e_{11} S_{n} e_{11}$. Consider the correspondence

$$
w: \sum x_{i} d_{i} \rightarrow \sum x_{i} v\left(d_{i}\right) \quad \text { for } x_{i} \in S_{n}, d_{i} \in D .
$$

If $\sum x_{i} d_{i}=0, \sum e_{1 j} x_{i} e_{11} d_{i}=e_{1 j} \sum x_{i} d_{i}=0$ for every $j$, and so $0=v\left(\sum e_{1 j} x_{i} e_{11} d_{i}\right)=\sum e_{1 j} x_{i} e_{11} v\left(d_{i}\right)=e_{1 j} \sum x_{i} v\left(d_{i}\right)$, whence $e_{j j} \sum x_{i} v\left(d_{i}\right)=0$ 
for every $j$. Thus, $\sum x_{i} v\left(d_{i}\right)=0$, which implies that $w$ is a homomorphism. Since $S_{n}$ is left self injective, $w$ is given by the right multiplication of an element $y \in S_{n}$. It follows then that the right multiplication of $e_{11} y e_{11}$ is an extension of $v$, proving the left self injectivity of $e_{11} S_{n} e_{11}(\simeq S)$. This completes the proof.

8.4. COROLLARY. Let $S$ be a ring. Then the following conditions are equivalent:

(1) $S$ is left self injective.

(2) The total matrix ring $S_{n}$ of degree $n$ is left continuous for some $n>1$.

(3) $S_{n}$ is left continuous for every $n$.

Proof. (1) implies (3) by Theorems 8.3 and 4.7. If we assume (2), $S_{n}$ for the $\boldsymbol{n}$ is left self injective by Corollary 7.5, and hence $S$ itself is also left self injective by Theorem 8.3 .

\section{REFERENCES}

1. C. W. Curtis and I. Reiner, Representation theory of finite groups and associative algebras, Interscience, New York, 1962.

2. N. Jacobson, Some remarks on one-sided inverses, Proc. Amer. Math. Soc. 1 (1950), 352-355.

3. - Structure of rings, Amer. Math. Soc. Colloq. Publ. Vol. 37, Ame:. Math. Soc., Providence, R.I., 1956.

4. R. E. Johnson, The extended centralizer of a ring over a module, Proc. Amer. Math. Soc. 2 (1951), 891-895.

5. J. von Neumann, Continuous geometry, Princeton Mathematical Series, No. 25, Princeton Univ. Press, Princeton, N. J., 1960.

6. Yuzo Utumi, On a theorem on modular lattices, Proc. Japan Acad. 35 (1959), 16-21.

7. - On continuous regular rings and semisimple self injective rings, Canad. J. Math. 12 (1960), 597-605.

8. - On continuous regular rings, Canad. Math. Bull. 4 (1961), 63-69.

\section{UNIVERSITY OF ROCHESTER,}

ROCHESTER, NEW YORK 